\title{
High-Order Mean-Field Approximations for Adaptive Susceptible-Infected-Susceptible Model in Finite-Size Networks
}

\author{
Kai Wang $\mathbb{D}^{1},{ }^{1}$ Xiao Fan Liu $\mathbb{D},^{2}$ and Dongchao Guo $\mathbb{D D}^{3}$ \\ ${ }^{1}$ School of Computer Science and Technology, Harbin Institute of Technology, Weihai 264209, China \\ ${ }^{2}$ Department of Media and Communication, City University of Hong Kong, Kowloon Tong, Hong Kong SAR, China \\ ${ }^{3}$ School of Computer Science, Beijing Information Science and Technology University, Beijing 100192, China
}

Correspondence should be addressed to Dongchao Guo; dongchaoguo@bistu.edu.cn

Received 3 October 2020; Revised 21 November 2020; Accepted 11 February 2021; Published 23 February 2021

Academic Editor: Honglei Xu

Copyright (c) 2021 Kai Wang et al. This is an open access article distributed under the Creative Commons Attribution License, which permits unrestricted use, distribution, and reproduction in any medium, provided the original work is properly cited.

Exact solutions of epidemic models are critical for identifying the severity and mitigation possibility for epidemics. However, solving complex models can be difficult when interfering conditions from the real-world are incorporated into the models. In this paper, we focus on the generally unsolvable adaptive susceptible-infected-susceptible (ASIS) epidemic model, a typical example of a class of epidemic models that characterize the complex interplays between the virus spread and network structural evolution. We propose two methods based on mean-field approximation, i.e., the first-order mean-field approximation (FOMFA) and higher-order mean-field approximation (HOMFA), to derive the exact solutions to ASIS models. Both methods demonstrate the capability of accurately approximating the metastable-state statistics of the model, such as the infection fraction and network density, with low computational cost. These methods are potentially powerful tools in understanding, mitigating, and controlling disease outbreaks and infodemics.

\section{Introduction}

Compartmental models, e.g., the SIS and SIR models, are the most commonly used frameworks to characterize the dynamics of ubiquitous diffusion processes such as epidemics [1], rumors, and computer network data flows [2]. Analytical solutions to these models using mean-field approximations can yield plenty of useful information regarding the severity and controllability of a diffusion process. For example, the finding of the absence of an epidemic threshold in scale-free networks has successfully raised the vigilance of the difficulty of controlling pandemic in large populations [3]. This paper addresses the mean-field approximation solution to epidemics in an adaptive and finite-size network.

It has been widely observed that the epidemic spreading and other network dynamics can have a complex interplay with the topology of the underlying network [4-7]. On the one hand, the epidemic propagates among nodes according to certain rules; on the other hand, the network topology also changes in response to the epidemic process. This phenomenon is also referred to as adaptive evolution. Gross et al. formulated the adaptive evolution of epidemic dynamics and network topology with a susceptible-infectedsusceptible model in infinite-size networks and revealed via homogeneous mean-field approximation that the system could have four possible steady states, namely, the healthy, endemic, oscillatory, and bistable states [4].

Although the adaptive evolution can exhibit a complex bifurcation pattern of system states in an infinite-size network, i.e., a network with an infinite number of nodes, for a finite-size network, the only steady state is when all nodes' states are converged, e.g., all healthy [8]. Therefore, the research focus of epidemics in finite-size networks is the "metastable" states, in which the system remains for a long time before getting into the absorbing state. Chakrabarti et al. [9] and Van Mieghem et al. [8, 10] derived the metastable-state infection fraction for the discrete-time and continuous-time epidemics, respectively, with mean-field approximations and found that the epidemic threshold of the finite-size network is upper-bounded by the largest eigenvalue of the adjacency matrix of the underlying network topology. 
Guo et al. $[11,12]$ proposed a general and exact adaptive susceptible-infected-susceptible model (ASIS) for finite-size networks. The model describes the exact Markovian process of the time-evolution characteristic of the system's viral state as a whole. This framework allows us, in principle, to derive the expressions of the metastable-state metrics (e.g., the infection fraction and the epidemic threshold). Unfortunately, without resorting to mean-field approximations, Guo et al. could only obtain implicit forms of the metastable-state metrics. Although their implicit expressions, to some extent, help to give insights into the complex interplay between the epidemic dynamics and the topological dynamics, they failed in isolating the dependent variables (i.e., the metrics) given the independent variable set (e.g., the infection rate, the recovery rate, and the adjacency matrix). It is thus intriguing to propose an approximation to the exact ASIS model, making it possible to calculate the value of the metastable-state metrics.

In this paper, we propose two expansions to mean-field methods, i.e., the first-order mean-field approximation and the higher-order mean-field approximation, and use them to derive exact solutions of metastable states of the ASIS epidemic process, i.e., the infection fraction and network density, and empirically verify the existence of the steady states of these approximations. We also examine the complex effects of the ASIS process parameters on the metastable state using these computable methods.

The rest of this paper is organized as follows. First, Section 2 elaborates on the SIS process in adaptive networks, followed by introducing the Markov theory-based ASIS model proposed previously [11]. Then, the two mean-field expansions for the exact ASIS model are presented in Section 3. Section 4 shows the results, i.e., derived metastable states, of the mean-field approximation methods and compares the results with simulations. The effects of the parameter settings of the ASIS process are also investigated. Section 5 concludes our works.

\section{The ASIS Model}

We consider a population in which an individual $i$ can be either in the state of infected $X_{i}(t)=1$ or susceptible $X_{i}(t)=0$ at time $t$, with probabilities $v_{i}(t)=\operatorname{Pr}\left[X_{i}(t)=1\right]$ and $1-v_{i}(t)=1-\operatorname{Pr}\left[X_{i}(t)=1\right]$, respectively. For a Bernoulli random variable, the relation $E\left[X_{i}\right]=\operatorname{Pr}\left[X_{i}\right]$ holds. The population is connected with an undirected and unweighted graph $G(N, L)$ composed of $N$ nodes and $L$ links. The concerned network is denoted by an adjacency matrix $A$ with elements $a_{i j}=1$ if node $i$ and $j$ are connected or $a_{i j}=0$ otherwise.

We consider four independent Poisson processes in the network, as shown in Figure 1. First, an infected node in the network can infect its susceptible neighbor with a rate $\beta$. Second, an infected node recovers (to the state of susceptible) with a rate $\delta$. Therefore, for each node $i$, the probability of its state changes with

$$
\frac{d}{d t} E\left[X_{i}\right]=E\left[-\delta X_{i}+\left(1-X_{i}\right) \beta \sum_{j=1, j \neq i}^{N} a_{i j} X_{j}\right] .
$$

Meanwhile, the network topology changes in response to the epidemic dynamics. Therefore, third, if there exists a link connecting an infected node $j$ and a susceptible node $i$, the node $i$ will cut off the link with a rate $\zeta$. Finally, two susceptible nodes tend to create a link between them at rate $\xi$ if there is no link connecting the two nodes. Besides, we denote by $\tau$ and $\omega$ the effective infection rate and the effective link-breaking rate. The adaptation of the network topology to the epidemic process is as follows:

$$
\begin{aligned}
\frac{d}{d t} E\left[a_{i j}\right] & =a_{i j}(0) E\left\{-\zeta a_{i j}\left[X_{i}\left(1-X_{j}\right)+X_{j}\left(1-X_{i}\right)\right]\right\}+\xi\left(1-a_{i j}\right)\left(1-X_{i}\right)\left(1-X_{j}\right) \\
& =a_{i j}(0) E\left[-\zeta a_{i j}\left(X_{i}-X_{j}\right)^{2}+\xi\left(1-a_{i j}\right)\left(1-X_{i}\right)\left(1-X_{j}\right)\right] .
\end{aligned}
$$

The link-breaking process $\omega$ mimics the immune strategy employed by the node $i$, and the link-creating process mimics the establishment of social ties among people.

The concerned topological metrics are introduced in the following. The metastable-state infection fraction is defined as follows:

$$
y_{\infty}=\lim _{t \longrightarrow \infty} \frac{\sum_{i=1}^{N} v i(t)}{N}=\frac{\sum_{i=1}^{N} v_{i \infty}}{N} .
$$

Another metric concerned in this paper is the normalized number of links (i.e., the network density) defined as follows:

$$
L_{\infty}=\lim _{t \rightarrow \infty} \frac{2 L}{N(N-1)}=\frac{\sum_{i} \sum_{j} a_{i j \infty}}{(N(N-1) / 2)}
$$

Under the above framework, the exact solutions to the infection fraction and the epidemic threshold could be derived (Theorem 1 and Theorem 2 in [11]). Analogously, other metastable-state metrics could be obtained. Although these expressions give insights into the complex interplay between the epidemic dynamics and the topological dynamics, they are unsolvable. To fill this gap, in the following, we will propose two solvable mean-field expansions to the ASIS model and investigate the accuracy of these approximations. 


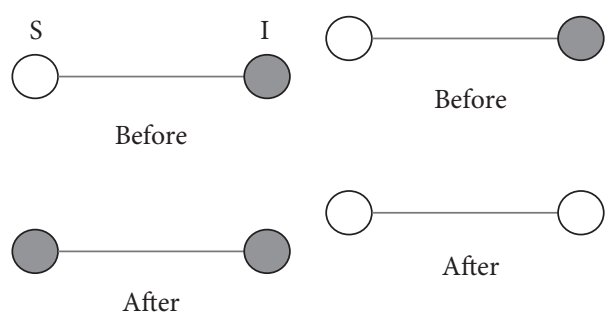

(a)

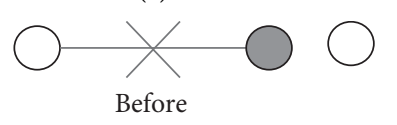

0

(b)

Before

Figure 1: Illustration of four independent epidemics and topology adaptation Poisson processes in the network.

\section{Mean-Field Approximations to the ASIS Model}

3.1. First-Order Mean-Field Approximation. The simplest mean-field expansion, which we name the first-order mean-field approximation (FOMFA), assumes that $E$ $\left[X_{i} a_{i j} X_{j}\right]=E\left[X_{i}\right] E\left[a_{i j}\right] E\left[X_{j}\right]$. Hence, the change of state for each node $i$ in equation (1) can be approximated to

$$
\frac{d}{d t} E\left[X_{i}\right]=-\delta E\left[X_{i}\right]+\left(1-E\left[X_{i}\right]\right) \times \beta\left\{\sum_{j=1}^{N} E\left[a_{i j}\right] E\left[X_{j}\right]\right\}
$$

and that the change of network topology in equation (2) can be approximated to

$$
\frac{d}{d t} E\left[a_{i j}\right]=-\zeta E\left[a_{i j}\right]\left(E\left[X_{i}\right]-E\left[X_{j}\right]\right)^{2}+\xi\left(1-E\left[a_{i j}\right]\right)\left(1-E\left[X_{i}\right]\right)\left(1-E\left[X_{j}\right]\right)
$$

Obviously, this approximation can be an oversimplified abstraction of the system, as the assumption of $E\left[X_{i} a_{i j} X_{j}\right]=E$ $\left[X_{i}\right] E\left[a_{i j}\right] E\left[X_{j}\right]$ implies that the nodes and link states are independent and therefore ignores the coupling of the triple term $\left[X_{i} a_{i j} X_{j}\right]$ into consideration. Nonetheless, the simplicity of this approximation still makes it a useful method to calculate the metastable-state infection fraction when the effective infection rate $\tau$ is relatively large at the expense of accuracy.

3.2. Higher-Order Mean-Field Approximation. To accurately capture the higher-order interactions between the network dynamics and topology, we consider the network evolution equation at a "node-link-node" triple level. In this case, the state of a triple can be one in the six possible states: SCS, SCI, $S D S, S D I, I C I$, and $I D I$, where $S$ and $I$ stand for nodes in the susceptible and infected states, respectively, and $C$ and $D$ stand for connected and disconnected links, respectively. Figure 2 illustrates the Markov state transition diagram among all the possible triple states. Note that eight states are shown in the figure, as for the SCI and SDI states, and the infected node can be either node in the triple.

The changes of the node state $E\left[X_{i}\right]$ and network connection $E\left[a_{i j}\right]$ and the "node-link-node" triple can be expressed as follows. Note that the change of node state is influenced by its recover rate and aggregated infectious effect from infected neighbors.

$$
\frac{d}{d t} E\left[X_{i}\right]=-\delta E\left[X_{i}\right]+\beta \sum_{j=1, j \neq i}^{N} E\left[\left(1-X_{i}\right) a_{i j} X_{j}\right] .
$$

The change of link state is influenced by

$$
\frac{d}{d t} E\left[a_{i j}\right]=-\zeta\left(E\left[X_{i} a_{i j}\left(1-X_{j}\right)\right]+E\left[\left(1-X_{i}\right) a_{i j} X_{j}\right]\right)+\xi E\left[\left(1-X_{i}\right)\left(1-a_{i j}\right)\left(1-X_{j}\right)\right] .
$$


The change of the probability of the triple SDS is as follows:

$$
\begin{aligned}
& \frac{d}{d t} E\left[X_{i}\left(1-a_{i j}\right) X_{j}\right]=-2 \delta E\left[X_{i}\left(1-a_{i j}\right) X_{j}\right]+E\left[\left(1-X_{i}\right)\left(1-a_{i j}\right) X_{j}\right] \\
& \quad \times \beta \sum_{k}\left(E\left[\left(1-X_{i}\right) a_{i k} X_{k}\right]+E\left[X_{i} a_{i k} X_{k}\right]\right)+E\left[\left(1-X_{j}\right)\left(1-a_{j i}\right) X_{i}\right] \times \beta \sum_{l}\left(E\left[\left(1-X_{j}\right) a_{j l} X_{l}\right]+E\left[X_{j} a_{j l} X_{l}\right]\right) .
\end{aligned}
$$
follows:

The change of the probability of the triple SCS is as

$$
\begin{aligned}
& \frac{d}{d t} E\left[X_{i} a_{i j} X_{j}\right]=-2 \delta E\left[X_{i} a_{i j} X_{j}\right]+E\left[\left(1-X_{i}\right) a_{i j} X_{j}\right] \beta \\
& \quad \sum_{k}\left(E\left[\left(1-X_{i}\right) a_{i k} X_{k}\right]+E\left[X_{i} a_{i k} X_{k}\right]\right)+E\left[\left(1-X_{j}\right) a_{j i} X_{i}\right] \beta \sum_{l}\left(E\left[\left(1-X_{j}\right) a_{j l} X_{l}\right]+E\left[X_{j} a_{j l} X_{l}\right]\right) .
\end{aligned}
$$

The change of the probability of the triple IDI is as follows:

$$
\begin{aligned}
& \frac{d}{d t} E\left[\left(1-X_{i}\right)\left(1-a_{i j}\right)\left(1-X_{j}\right)\right]=-E\left[\left(1-X_{i}\right)\left(1-a_{i j}\right)\left(1-X_{j}\right)\right] \\
& \quad \times\left\{\xi+\beta \sum_{k}\left(E\left[\left(1-X_{i}\right) a_{i k} X_{k}\right]+E\left[X_{i} a_{i k} X_{k}\right]\right)+\beta \sum_{l}\left(E\left[\left(1-X_{j}\right) a_{j l} X_{l}\right]+E\left[X_{j} a_{j l} X_{l}\right]\right)\right\}+\delta\left\{E\left[\left(1-X_{i}\right)\left(1-a_{i j}\right) X_{j}\right]+E\left[\left(1-X_{j}\right)\left(1-a_{j i}\right) X_{i}\right]\right\} .
\end{aligned}
$$

The change of the probability of the triple ICI follows:

$$
\begin{aligned}
& \frac{d}{d t} E\left[\left(1-X_{i}\right) a_{i j}\left(1-X_{j}\right)\right]=-E\left[\left(1-X_{i}\right) a_{i j}\left(1-X_{j}\right)\right] \\
& \quad \times\left\{\beta \sum_{k}\left(E\left[\left(1-X_{i}\right) a_{i k} X_{k}\right]+E\left[X_{i} a_{i k} X_{k}\right]\right)+\beta \sum_{l}\left(E\left[\left(1-X_{j}\right) a_{j l} X_{l}\right]+E\left[X_{j} a_{j l} X_{l}\right]\right)\right\}+\delta\left\{E\left[\left(1-X_{i}\right) a_{i j} X_{j}\right]+E\left[\left(1-X_{j}\right) a_{j i} X_{i}\right]\right\}+\xi E\left[\left(1-X_{i}\right)\left(1-a_{i j}\right)\left(1-X_{j}\right)\right] .
\end{aligned}
$$

The change of the probability of the triple SDI is as follows:

$$
\begin{aligned}
& \frac{d}{d t} E\left[\left(1-X_{i}\right)\left(1-a_{i j}\right) X_{j}\right]=-E\left[\left(1-X_{i}\right)\left(1-a_{i j}\right) X_{j}\right] \\
& \quad \times\left\{\beta \sum_{k}\left(E\left[\left(1-X_{i}\right) a_{i k} X_{k}\right]+E\left[X_{i} a_{i k} X_{k}\right]\right)+\delta\right\}+\delta E\left[X_{i}\left(1-a_{i j}\right) X_{j}\right] \\
& \quad+E\left[\left(1-X_{i}\right)\left(1-a_{i j}\right)\left(1-X_{j}\right)\right] \times \beta \sum_{l}\left(E\left[\left(1-X_{j}\right) a_{j l} X_{l}\right]+E\left[X_{j} a_{j l} X_{l}\right]\right)+\zeta E\left[\left(1-X_{i}\right) a_{i j} X_{j}\right] .
\end{aligned}
$$


follows:

The change of the probability of the triple SCI is as

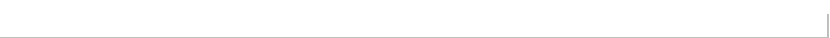

$$
\begin{aligned}
\frac{d}{d t} E\left[\left(1-X_{i}\right) a_{i j} X_{j}\right]= & -E\left[\left(1-X_{i}\right) a_{i j} X_{j}\right] \times\left\{\zeta+\delta+\beta \sum_{k}\left(E\left[\left(1-X_{i}\right) a_{i k} X_{k}\right]+E\left[X_{i} a_{i k} X_{k}\right]\right)\right\} \\
& +\delta E\left[X_{i} a_{i j} X_{j}\right]+E\left[\left(1-X_{i}\right) a_{i j}\left(1-X_{j}\right)\right] \times\left\{\beta \sum_{l}\left(E\left[\left(1-X_{j}\right) a_{j l} X_{l}\right]+E\left[X_{j} a_{j l} X_{l}\right]\right)\right\} .
\end{aligned}
$$

The above governing equations (7)-(14) are named the high-order mean-field approximation (HOMFA). From HOMFA, the steady-state values of all the variables as well as the metastable-state metrics such as the infection fraction $y$ are defined in equation (3).

\section{Simulations and Numerical Results}

4.1. The Metastable State and Simulation Method. One could denote the system state by the vector of $\mathrm{N}$ components, where $i$-th component represents the viral state of $v_{i}$. Each possible value of the vector represents a specific system state. The state space composed of all possible states is finite for the SIS process in finite-size networks. Usually, the system state could change from one to another following a finite Markovian process. We name the particular state, which the system could change into but not vice versa, as the absorbing state. Van Mieghem et al. noted that the only final steady state of the exact Markov process representing the SIS process in a finite-size network is an absorbing state where all nodes are healthy [8]. Before reaching this absorbing state, the exact SIS model remains for a long time in a "metastable state." Generally speaking, it is a bit hard to precisely determine the metastable state partly because it depends not only on the network size but also on other parameters of the SIS model. Take, for example, the classical SIS process model in finite-size networks, it is possible to experimentally determine the metastable state and calculate some statistical metrics such as the metastable-state infection fraction [13] although the metastable state is not defined precisely. In order to get the average value of a metastable-state metric for a specific configuration of parameters, one could run a number of simulation instances and compute the value of the metric over time and look for a plateau. Then, one should determine empirically a time point at the plateau and specify the value at the time point as the metastable-state value [9]. Obviously, this approach looks inaccurate as a simulation method.

Alternatively, one could change the exact SIS model slightly by introducing a nodal self-infection Poisson process with rate $\varepsilon$ [14], which can be seen as a perturbation to the exact SIS model. Specifically, the self-infection process enables each node to spontaneously become infected. The introduction of the selfinfection process means that the state where all nodes are healthy is no more the absorbing state. In other words, the absorbing state is eliminated from the state space because the probability of leaving the all healthy state is slightly larger than zero for small $\varepsilon$. As a result, one will not be bothered to determine empirically the metastable state. Instead, one could run only one simulation instance for a long time while recording the change in the value of the concerned metric. Then, one only needs to calculate the time-average value of the metric which is also named as the steady-state value. The steady-state value of the $\varepsilon$-SIS offers a well approximation to the metastable-state value of the corresponding exact SIS model [13].

Extending the $\varepsilon$-SIS model elaborated above by introducing the link dynamics, Guo et al. proposed the $\varepsilon$-ASIS model and also showed that the "metastable state" of the exact ASIS model could be accurately approximated by the steady state of the corresponding adaptive $\varepsilon$-SIS simulation approach (Section 3 in [11]). We implement an event-driven time-continuous $\varepsilon$-SIS simulator to calculate the metastable-state statistical metrics of the exact ASIS process. One apparent advantage of the $\varepsilon$-SIS simulator is that it will not bother to determine whether and when the system enters into the metastable state, which is not clearly defined. One only needs to choose a proper small $\varepsilon$ and to run the simulator for a warm-up period. Then, one starts to record the change of the concerned metric, based on which the steady-state value of the metric could be calculated over the whole recording period. If the recording period is long enough, the steady-state value is considered accurately approximate to the metastable-state value.

Figure 3 demonstrates the comparisons between the exact ASIS process and HOMFA with respect to the changes in the infection fraction and the number of links. The exact ASIS process is run with a nontrivial configuration of parameters: a complete graph with the number of nodes $N=40, \delta=1, \zeta=1, \xi=1$, and $\varepsilon=10^{-3}$. The solid lines stand for the changes of the metrics concerned, while the dashed lines represent the HOMFA expansion. It shows that there exists a metastable-state of the exact ASIS process. After a sharp change, both metrics' values found some new level of equilibrium for the period of the observing time. The proposed mean-field approximations HOMFA can approximate the exact ASIS process accurately.

4.2. Comparisons between FOMFA and HOMFA. We compare the metastable-state infection fraction and the normalized number of the two proposed mean-field approximations, i.e., FOMFA and HOMFA. The metrics of the exact ASIS process are empirically obtained via the simulations of the corresponding $\varepsilon$-ASIS model depicted in Section 4.1. The metrics of the mean-field approximations are numerically calculated. As shown in Figure 4, 


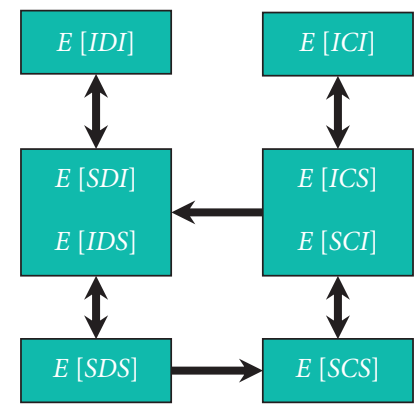

FIGURE 2: State transition diagram in the proposed high-order mean-field approximation.

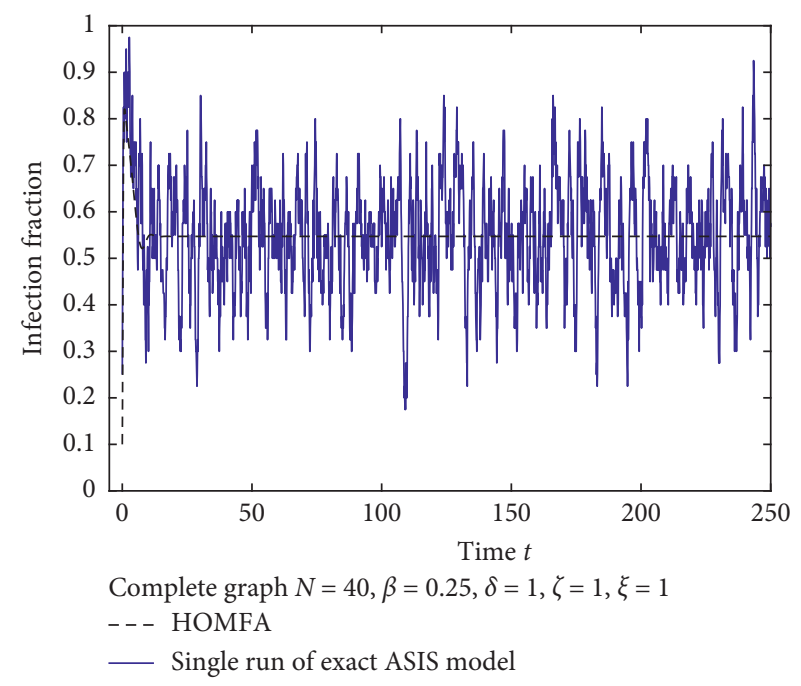

(a)

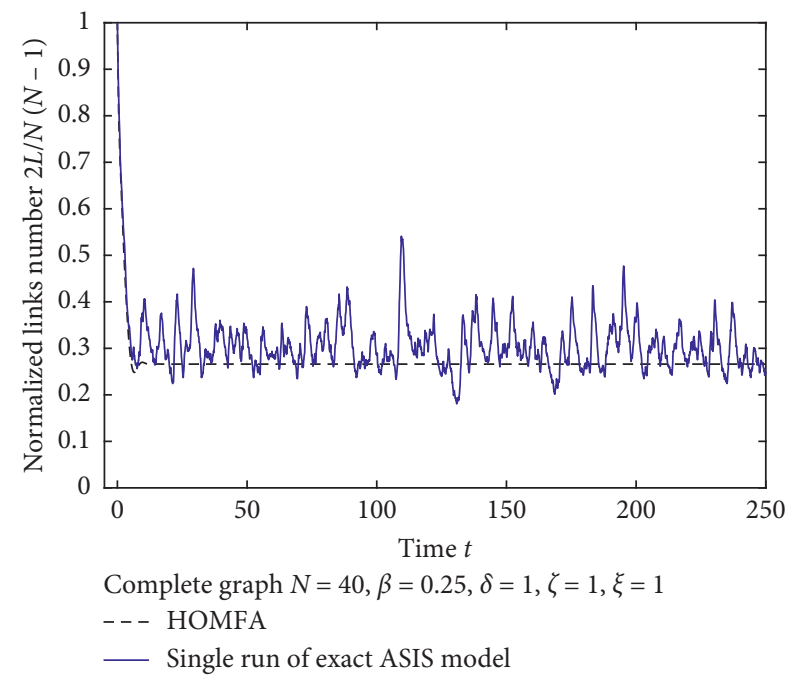

(b)

FIgURE 3: The time evolution of the adaptive SIS process in the complete graph for the configuration $N=40, \delta=1, \zeta=1, \xi=1$, and $\varepsilon=10^{-3}$. The black dashed line denotes the high-order mean-field approximation (HOMFA). The blue line denotes one single run of the exact ASIS process. Two metastable-state metrics, namely, the infection fraction and the normalized number of links, are shown.

both approximations can closely reproduce the metastable-state metrics. Also noticeable is that both approximations perform well as the effective infection rate $\tau$ increases, given that the other parameters are fixed. As the effective infection rate increases, more nodes are in the infected state so that there are more connections between the infected nodes and the susceptible nodes. As a result, there would be more links getting broken because of the link-breaking process. Both the mean-field approximations and the exact ASIS process show the decline in the number of links. However, the HOMFA performs a bit better than the FOMFA. If more accurate predictions are needed, the HOMFA may be the better choice. Nevertheless, it would not be ignored that better accuracy is achieved at the expense of much higher computational complexity.

We also investigate the impact of both the infection rate and link-breaking rate on the metastable-state infection fraction. As shown in Figure 5, the increase in the infection rate and the decrease in the link-breaking rate can increase the infection fraction in the metastable state. For a fixed effective link-breaking rate, the infection fraction increases rapidly as the effective infection rate rises. In contrast, the increase in the link-breaking rate leads to a minor decrease in the infection fraction for a fixed infection rate. These observations imply that the infection rate's impact is much higher than that of the link-breaking rate. 


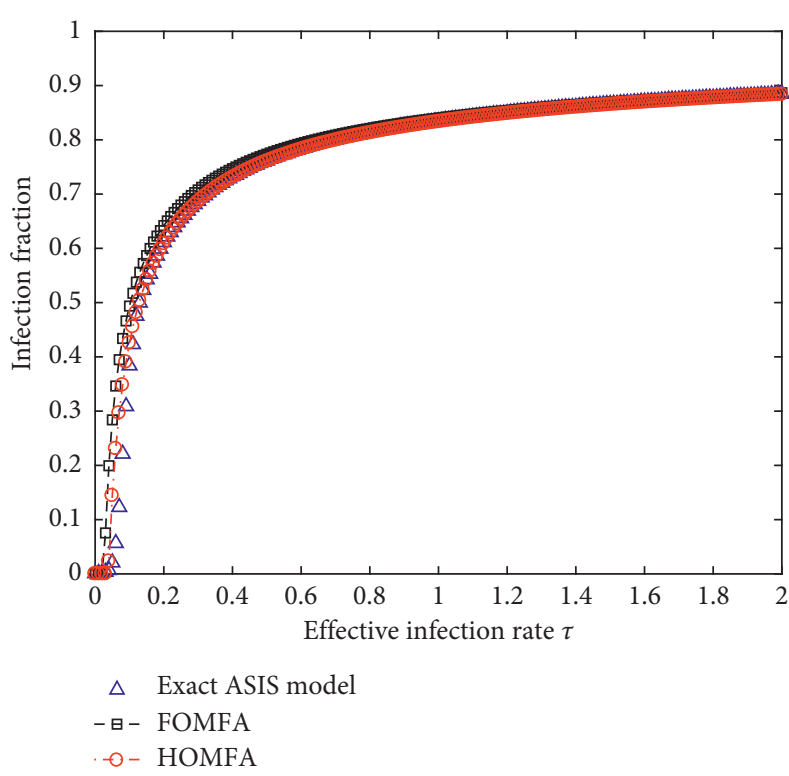

(a)

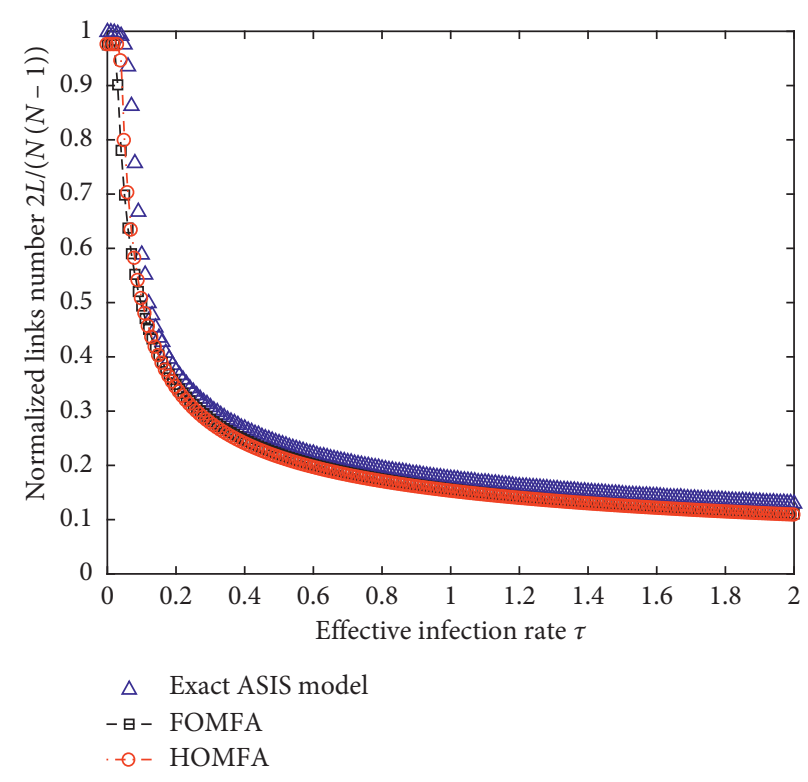

(b)

FIGURE 4: Comparison between the mean-field approximations and the simulation results in complete graphs for $N=40, \delta=1, \zeta=1, \xi=1$, and $\varepsilon=10^{-3}$. The black dashed line denotes the first-order mean-field approximation (FOMFA) while the red dashed-dotted line denotes the high-order mean-field approximation (HOMFA). The blue triangles denote the simulation results. The impact of the effective infection rate and the effective link-breaking rate..

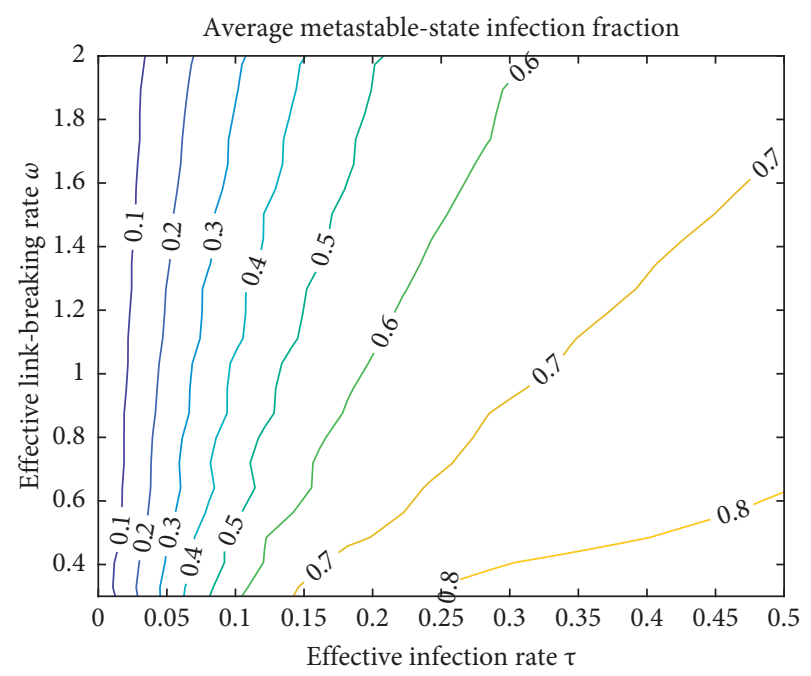

FIGURE 5: The average metastable-state infection fraction as a function of the effective infection rate and the effective link-breaking rate.

\section{Conclusion}

The exact ASIS model helps provide physical insights into the metastable-state infection fraction and the topology. Nevertheless, the expressions for the infection fraction and the epidemic threshold are unsolvable. As a result, they have difficulties applying to characterizing the metastable state of the ASIS process quantitatively and accurately. This paper presents two mean-field expansions to the exact ASIS model, namely, the first-order meanfield approximation (FOMFA) and the high-order mean- field approximation (HOMFA). We first present the governing equations for FOMFA and HOMFA. Based on these approximations, we could calculate the metastablestate infection fraction and the normalized number of links, which are the appropriate metrics for measuring the epidemic dynamics and the topology dynamics. The simulations show that the proposed approximations could approximate the exact ASIS process in relative accuracy. Under the approximation framework, the pattern of the impact of the infection rate and the link-breaking rate on the metastable-state infection fractions is also investigated. Our works provide a computable tool for those focusing on the virus spread in adaptive networks. For example, the mean-field approximations help better understand the complex impact of the parameters on the epidemic threshold [15].

Furthermore, our works help individuals to design better quarantine or immune strategies [1,16-21]. The theoretical approach for epidemic modelling employed by the ASIS model is an example of the individual-based mean-field approach [1], which can be extended to formalize the individual's dilemma in optimally allocating containment resources (e.g., vaccines and social distancing) to suppress the disease while minimizing the cost. The cost is usually consisted of the payment of resource usage and the adverse effect of being infected. The decrease in the payment of resource usage will lead to the increase in the payment of being infected, and vice versa. It is straightforward to model, formulize, and solve this dilemma under the framework of optimization technique [14]. Therefore, based on the meanfield approximations proposed in this paper, the effect of strategies could be assessed quantitatively. 


\section{Data Availability}

No data were used to support this study.

\section{Conflicts of Interest}

The authors declare that they have no conflicts of interest regarding the publication of this paper.

\section{Acknowledgments}

This research was supported by the National Natural Science Foundation of China (NSFC) (grant no. 61702439) and the Double First-Class Scientific Research Funds of HIT (grant no. IDGA1010200107).

\section{References}

[1] R. Pastor-Satorras, C. Castellano, P. V. Mieghem, and A. Vespignani, "Epidemic processes in complex networks," Review of Modern Physics, vol. 87, no. 3, pp. 925-979, 2015.

[2] W. Yang, Y. Qin, and Y. Yang, "Analysis of malicious flows via SIS epidemic model in CCN," in Proceedings of the 2018 - IEEE Conference on Computer Communications Workshops (INFOCOM WKSHPS), pp. 748-753, Honolulu, HI, USA, April 2018.

[3] R. Pastor-Satorras and A. Vespignani, "Epidemic spreading in scale-free networks," Physical Review Letters, vol. 86, no. 14, pp. 3200-3203, 2001.

[4] T. Gross, C. D’Lima, and B. Blasius, "Epidemic dynamics on an adaptive network," Physical Review Letters, vol. 96, no. 20, pp. 1-4, 2006.

[5] L. D. Valdez, P. A. Macri, and L. A. Braunstein, "Intermittent social distancing strategy for epidemic control," Physical Review E, vol. 85, no. 3 Pt 2, pp. 1-7, 2012.

[6] N. Masuda and P. Holme, Temporal Network Epidemiology, Springer Singapore, Singapore, 2017.

[7] S. Boccaletti, G. Bianconi, R. Criado et al., "The structure and dynamics of multilayer networks," Physics Reports, vol. 544, no. 1, pp. 1-122, 2014.

[8] P. V. Mieghem, J. Omic, and R. Kooij, "Virus spread in networks," IEEE/ACM Transactions on Networking, vol. 17, no. 1, pp. 1-14, 2009.

[9] D. Chakrabarti, Y. Wang, C. Wang, J. Leskovec, and C. Faloutsos, "Epidemic thresholds in real networks," ACM Transactions on Information and System Security, vol. 10, no. 4, pp. 1-26, 2008.

[10] E. Cator and P. V. Mieghem, "Second-order mean-field susceptible-infected-susceptible epidemic threshold," Physical Review E, vol. 85, no. 5 Pt 2, pp. 1-7, 2012.

[11] D. Guo, S. Trajanovski, R. Bovenkamp, H. Wang, and P. V. Mieghem, "Epidemic threshold and topological structure of susceptible-infectious-susceptible epidemics in adaptive networks," Physical Review E, vol. 88, no. 4, pp. 1-14, 2013.

[12] S. Trajanovski, D. Guo, and P. V. Mieghem, "From epidemics to information propagation: striking differences in structurally similar adaptive network models," Physical Review E, vol. 92, no. 3, pp. 1-5, 2015.

[13] C. Li, R. Bovenkamp, and P. V. Mieghem, "Susceptibleinfected-susceptible model: a comparison of n-intertwined and heterogeneous mean-field approximations," Physical Review E, vol. 86, no. 2 Pt 2, pp. 1-9, 2012.
[14] P. Van Mieghem and E. Cator, "Epidemics in networks with nodal self-infection and the epidemic threshold," Physical Review E, vol. 86, no. 1 Pt 1, pp. 1-10, 2012.

[15] Z. Chen, "Epidemic thresholds in networks: impact of heterogeneous infection rates and recovery rates," in Proceedings of the IEEE International Conference on Communications (ICC), pp. 1-6, Kansas City, MO, USA, May 2018.

[16] S. Trajanovski, Y. Hayel, E. Altman, H. Wang, and P. V. Mieghem, "Decentralized protection strategies against SIS epidemics in networks," IEEE Transactions on Control of Network Systems, vol. 2, no. 4, pp. 406-419, 2015.

[17] C. Nowzari, V. M. Preciado, and G. J. Pappas, "Analysis and control of epidemics: a survey of spreading processes on complex networks," IEEE Control Systems Magazine, vol. 36, no. 1, pp. 26-46, 2016.

[18] J. Wang, W. Wang, and C. Wang, "Divide and conquer: leveraging topology in control of epidemic information dynamics," in Proceedings of the 2016 IEEE Global Communications Conference (GLOBECOM), pp. 1-6, Washington, DC, USA, December 2016.

[19] A. Laszka, M. Felegyhazi, and L. Buttyan, "A survey of interdependent information security games," ACM Computing Surveys, vol. 47, no. 2, pp. 1-38, 2014.

[20] V. Marbukh, "Economics of networked infrastructures at the edge of undesirable contagion: a case of SIS infection," in Proceedings of the 2018 - IEEE Conference on Computer Communications Workshops (INFOCOM WKSHPS), pp. 1-2, Honolulu, HI, USA, April 2018.

[21] M. Ogura and V. M. Preciado, "Optimal containment of epidemics in temporal and adaptive networks," in Temporal Network Epidemiology, N. Masuda and P. Holme, Eds., pp. 241-266, Springer, Singapore, 2017. 